\title{
Spatial Temporal Modeling of Hospitalizations for Fall Related Hip Fractures in Older People
}

Robin M Turner, $\mathrm{PhD},{ }^{1}$ Andrew Hayen, $\mathrm{PhD},{ }^{1,2}$ William T M Dunsmuir, $\mathrm{PhD},{ }^{2}$ and Caroline F Finch, $\mathrm{PhD}^{1,3}$

\footnotetext{
${ }^{1}$ NSW Injury Risk Research Management Centre, University of New South Wales, Sydney NSW 2052, Australia, ${ }^{2}$ School of Mathematics and Statistics, University of New South Wales, Sydney NSW 2052, Australia, ${ }^{3}$ School of Human Movement and Sports Science, University of Ballarat, Ballarat Vic 3353, Australia
}

\section{Corresponding Author:}

Caroline Finch, School of Human Movement \& Sport Sciences, University of Ballarat PO Box 663, Ballarat Victoria 3353, Australia, +61 35327 9878, Fax, c.finch@ballarat.edu.au

\section{Mini-Abstract:}

The study determined the spatial temporal characteristics of fall related hip fractures in the elderly using routinely collected injury hospitalization and sociodemographic data. There was significant spatial temporal variation in hospitalized hip fracture rates in New South Wales, Australia. 


\begin{abstract}
Introduction: The study determined the spatial temporal characteristics of fall related hip fractures in the elderly using routinely collected injury hospitalization data.
\end{abstract}

Methods: All New South Wales (NSW), Australia, residents aged 65+ years who were hospitalized for a fall related hip fracture between 1 July 1998 and 30 June 2004, were included. Bayesian Poisson regression was used to model rates in local government areas (LGAs) allowing for the incorporation of spatial, temporal and covariate effects.

Results: Hip fracture rates were significantly decreasing in one LGA and there were no significant increases in any LGAs. The proportion of the population in residential aged care facilities was significantly associated with the rate of hospitalized hip fractures with a relative risk (RR) of 1.003 (95\% Credible Interval 1.002, 1.004). Socioeconomic status was also related to hospitalized hip fractures with those in the third and fourth quintiles being at decreased risk of hip fracture compared to those in the least disadvantaged (fifth) quintile $(\mathrm{RR}=0.837(0.717,0.972)$ and $\mathrm{RR}=0.855(0.743,0.989)$ respectively).

Conclusions: There was significant spatial temporal variation in hospitalized hip fracture rates in NSW, Australia. The use of Bayesian methods was crucial to allow for spatial correlation, covariate effects and LGA boundary changes.

Key Words: Bayesian, hip fractures, older people, Poisson regression, Spatial Temporal Modeling. 


\section{INTRODUCTION}

Falls and their resultant injuries place a substantial burden on older individuals, because of loss of their quality of life, and on society, because of the costs of acute health care provision and long-term rehabilitation. Hip fractures are one of the most common, and costly, falls related injury in older people [1]. While trends in hip fracture rates in the elderly have largely stabilised internationally [1, 2, 3, 4, 5], the number of cases is increasing in developed countries because of the ageing population and the overall burden associated with such injuries is likely to increase further over the next few years[1]. It is also likely that there will be an increasingly differential burden in different regions due to social-demographic variation and health service delivery factors. This will necessitate the targeting of local level interventions to address these inequalities. For this reason, it is becoming increasingly important for injury statistics to be calculated and presented at a local or regional level (i.e. spatially).

Currently, there is limited data on spatial trends in fall-related hip fractures internationally. While temporal, or time, trends are well understood [1, 2, 3, 4, 5], only one study has examined spatial variation in hip fractures [6]. That study, conducted in Alberta, Canada, considered the number of emergency department presentations after a fall and showed spatial variation and significant clustering of cases by place of residence [6].

Some important social-demographic characteristics have been identified at the population level for hip fracture or fall rates. The hip fracture rate remains much higher in women than men and increases with age [1]. Although socio-economic status may play some role in the risk of hip fracture at the individual level [7], at the population level there is little evidence 
that socio-economic status is related to hip fracture rates [8], although there is some evidence of a relationship with fall rates [8]. The type of residence has been shown to impact on hip fracture risk, with older people living in residential/nursing homes being at greater risk of hip fracture than those living in the community, even after adjustment for age and sex [9].

In order to accurately investigate the characteristics of population hip fracture rates at both a spatial and temporal level, appropriate statistical analysis methods need to model geographic areas with very few counts and allow the incorporation of geographic information to correctly specify relationships between neighbouring regions. This is important because the characteristics of adjacent areas, for example, are more likely to be similar than those separated by hundreds of kilometres. Furthermore, regional boundaries are somewhat arbitrary in that people who live close to such boundaries may seek health care on the basis of what is currently available to them, rather than on the basis of where they live. Importantly, statistical models must also be able to adjust for the age and sex structure of a population as well as other covariates of interest, such as socio-economic status. Appropriate methods to achieve this have seldom been used in injury epidemiology, although some studies of road safety and accidental poisoning research have begun to use these methods recently [10, 11, 12].

This paper presents an analysis of the spatial and temporal characteristics of hip fracture hospitalization rates in New South Wales (NSW), Australia after adjustment for age, gender and other population demographics. To our knowledge, there has not previously been a concurrent assessment of both the spatial and temporal characteristics as they relate to fall injuries or hip fracture hospitalizations rates in the international literature. The relationship between hip fracture hospitalization rates and key variables, such as socio-economic status 
and type of residence, is also investigated. This paper describes the modeling process in detail and justifies why this approach is necessary in the hope that future studies may also adopt this methodology.

\section{MATERIALS AND METHODS}

Data were obtained from the NSW Inpatient Statistics Collection (ISC), a financial year census of all inpatient hospital separations in New South Wales, for the six-year period 1 July 1998 to 30 June 2004, inclusive. To obtain a dataset based on admission date rather than separation date, we examined hospitalizations for which the date of separation was up to one year later to ensure that all admissions occurring on or before 30 June 2004 were obtained. Separations occur when an episode of inpatient hospital care is completed, that is, when a patient is discharged, transferred, or dies, or has a type-change separation (e.g. when the patient changes from an acute to a rehabilitation patient). Unfortunately, there is no unique patient identifier in the ISC, so we have attempted to eliminate multiple counting of hip fracture separations by excluding admissions for which the source of referral was a transfer from another hospital, or for a type change admission.

Cases were selected with a principal International Classification of Diseases (Australian Modification) (ICD-10-AM) diagnosis code of hip fracture (S72.0-S72.2) and the first external code of a fall (W00-W19). Only those aged 65+ years at the time of admission were included.

Population estimates by sex and statistical local area for people aged 65+ years were obtained from the Australian Bureau of Statistics (ABS) for local government areas (LGAs) in NSW 
using the 2001 boundaries [13]. The LGA boundaries did not remain constant across the study time frame and, without geocoded data, it is possible that any identified changes in rates in particular LGAs could be due to a boundary change rather than a 'true' change in the hospitalized hip fracture rate. To account for this in the model, all boundary changes were modelled using a simple step function at the time the boundary change occurred.

Information on the socioeconomic status of each LGA was obtained from the ABS. Socioeconomic status was measured using the Index of Relative Socioeconomic Disadvantage (IRSD), which combines information from the 2001 Australian census on income, unemployment and education for an area. The population, grouped into LGAs, was ranked into quintiles according to the IRSD scores. The first quintile contains approximately one fifth of the population and is the most disadvantaged LGAs; quintile five is the least disadvantaged LGAs.

The number of permanent residents by age (5 year age groups) and sex in residential aged care services, including facilities run by the government, not-for-profit organisations and private companies, was obtained from the Australian Government Department of Health and Ageing for people aged 65+ years.

To account for the differences in the age (using 5 year age groups) and sex structure across LGAs, we indirectly standardised rates to obtain standardised admission ratios (SAR) for each LGA using the rate for the whole of NSW as the standard. This means that the final rate estimates are age and sex adjusted, but that the different effects of these two factors could not be assessed separately. 
The standardised admission ratio $\left(\theta_{i j}\right)$ is given by

$$
\theta_{i j}=\frac{Y_{i j}}{E_{i j}}
$$

where $Y_{i j}$ is the observed count and $E_{i j}$ is the expected count in the $i$ th LGA at the $j$ th year. However, because estimates of standardised admission ratios may be unstable because of small populations and small numbers of hip fractures, we fitted Bayesian models to the data. The model that we fitted takes into account spatial correlation in rates, because rates in neighbouring areas tend to be more similar than in non-neighbouring areas. We also allowed for changes of boundaries in local government areas, temporal effects, and for other covariates, such as socio-economic status and the number of residents in residential aged care services in each LGA. Full details may be found in the statistical appendix.

It was hypothesised that there would be spatial variation in the hospitalized hip fracture rate over and above differences in the age and sex structure of the population, and that the rate would not be related to socio-economic status of the LGA. Additionally, it was considered, a priori, that LGAs with a greater proportion of people in aged-care facilities would have a higher hospitalized hip fracture rate.

The model, as described above, was fitted to the NSW hip fracture hospitalizations data and the hypotheses tested by examination of the model parameters. Estimates of precision of the model parameters, are given as 95\% Credible Intervals [15].

\section{RESULTS}


Overall, there were significant spatial and temporal effects. In general, hospitalized hip fracture rates were lower in the north east of the state and higher in parts of the west and southwest. By allowing the time variable to vary spatially by LGA, one LGA had a significant linear trend over time, the LGA of Ryde (in north-western Sydney) with a rate ratio of 0.969 (95\% credible interval 0.937 to 0.999$)$ per year, indicating a declining rate.

Figure 1 shows the standardised admission ratios, adjusted for the boundary changes, across NSW for hip fracture hospitalizations. The lowest rates are shown in white and the highest rates in black. Only data for the final year of the study, 2003-04, are shown but these are indicative of patterns in all of the other years examined. Figure 2 shows the corresponding graph for the Sydney metropolitan LGAs separately to highlight the most densely populated region in New South Wales. The Sydney region can be seen in Figure 1 as the densely grouped LGAs in the middle of the eastern coastline. In these two figures, some of the rate ratios are not significantly different from the overall NSW hip fracture hospitalization rate; the significantly high rate ratios are shown in Figures 3 and 4 . These figures show a high rate in the Unincorporated Far West/Broken Hill LGA, as well as a group of LGAs in the southwest region of New South Wales with higher rates than the state average.

Table 1 shows the (exponentiated) parameter estimates for the covariates of interest, these are adjusted for all other covariates in the model. The residential aged care facility variable is a measure of the number of people in residential aged care facilities in each LGA as a rate per 1000 population aged 65+ years. There was a significant increase in the rate of hospitalized hip fracture as the number of older people in residential aged care facilities increased. The interpretation of this is that, for every extra person per 1000 aged 65+ years in a residential 
aged care facility in a particular LGA, the LGA is 1.003 times more likely to have a hospitalized hip fracture of an elderly person.

The socio economic status variable (Table 1) measure quintiles of disadvantage with SES quintile 1 the most disadvantaged and SES quintile 5 the least disadvantaged reference group. The SARs show that, relative to the quintile of least disadvantage (quintile 5), SES quintile 3 and SES quintile 4 have significantly lower rates, in other words the least deprived quintile have a higher hip fracture rate than other the quintiles and this is significantly higher than those in SES quintile 3 and SES quintile 4.

Table 2 shows the SARs for the LGAs with the highest and lowest SARs. Sydney LGA has the highest rate with more than one and a half times as many hospitalized hip fractures compared to the overall state average. Richmond Valley has less than half as many hospitalized hip fractures compared to the overall state average.

The Sydney LGA had a boundary change occur between the 02/03 and 03/04 financial years gaining over 200 hectares from two surrounding LGAs, this was a densely populated region and equates to a substantial number of people. If the boundary change is ignored Sydney LGA had an SMR of 1.551 in the 2002/03 financial year, which jumps to 2.362 in the 03/04 financial year, after adjustment for the boundary change this rate ratio reduces to 1.669, which fits with the small non-significant increase in the SMR with time for the previous years.

The Sydney LGA, which has the highest standardised admission ratio, have on average an extra 3.8 hip fractures per 1000 people per year compared to the NSW average overall and an 
extra 7.6 hip fractures per 1000 people per year compared to those in the LGA with the lowest standardised admission ratio, Richmond Valley. The covariate effects are much smaller, for those in the 3rd and 4th socio-economic quintiles; they have an average of 0.9 and 0.8 hip fractures per 1000 people per year less than those in the 5th quintile (the least disadvantaged). The residential aged care facility effect equates to an extra 0.02 hip fractures per 1000 people per year.

\section{DISCUSSION}

This is the first study to examine the spatial temporal epidemiology of hospitalized hip fracture rates within a well-defined geographic region in the international peer-reviewed literature. The study considered the rate of hospitalization for fall-related hip fracture in New South Wales for people aged 65+ years across LGAs. We found that a number of LGAs had hip fracture hospitalization rates that were significantly different from the overall New South Wales rate for 65+ year olds, even after adjusting for differences in the age and sex profile in those regions. Unlike other falls-related fractures in older people, the majority of hip fractures are admitted to hospital [16]. This means that it is highly unlikely that the observed differences in rates across LGAs are due to different admission practices by hospitals across the state.

Importantly, we found that the least socioeconomically deprived areas had slightly higher rates of hip fractures than the more deprived areas. A previous study in the United Kingdom found no relationship between socioeconomic status and hip fracture rates [8]. We also found that regional hospitalized hip fracture rates increased with the number of people in residential aged care facilities in the region. This population level finding provides support 
to the previous individual-level studies, which have shown an increased incidence of hip fracture in nursing home residents compared to community-dwelling older people [17].

Our study used modern Bayesian statistical methods to describe the spatial distribution of hip fracture in NSW. These methods largely overcome problems with traditional methods (such as standardised mortality/admissions ratios), which are too variable and do not allow for spatial correlation in the data. Incorporating spatial correlation into the model leads to more robust estimates of the standardised admission ratios in each LGA and uses the surrounding LGAs so smooth out the extra variation in LGAs with low numbers. This method also allowed us to assess the time trend in each LGA; this would not be possible using traditional methods. Assessment of the time trend showed that only one LGA had a significant linear decrease in hospitalized hip fracture rates with time, no LGA had a significant increase in rates with time.

Adjustment for the boundary changes was important in the model to correct for possible misclassification of the hospitalized hip fractures to the underlying population. Without this adjustment, apparent increases or decreases in the rate due to the boundary change would have been found for some LGAs, with the most extreme case a large increase in the 2003/2004 financial year for the Sydney LGA that was associated with the boundary change rather than a real increase in hospitalized hip fractures.

There are a number of limitations to this study. Because NSW hospitalization data does not have a unique patient identifier and does not record the date of injury, we attempted to eliminate multiple counting by eliminating admissions that were recorded as being transfers or type change admissions. These exclusion criteria were chosen based on sensitivity and 
specificity after comparison with a linked hospital to hospital dataset where first admissions for a hip fracture could be identified (see Appendix). The linked data could not be used for the analysis because it was only available for a subset of the time period. However, despite these efforts, it is possible that we have not estimated the true incidence of hospitalized hip fracture by LGA. Multiple counting is more likely to affect the LGAs with small populations where people need to be transferred to larger hospitals for treatment. We would then expect that relative risks in the western part of New South Wales, with small LGAs, could be overestimated.

Because of the data available in the ISC, we were not able to measure or adjust for other potential factors that might affect hip fracture incidence such as comorbidities, medication use and frailty $[1,18,19]$. However, our modeling approach did adjust for the two consistently most powerful risk factors of age and sex. Our significant finding of a spatial relationship with the number of residential aged care facilities in a region, confirms earlier research that the type of residence for older people, impacts on their hip fracture rates [20].

We have looked at socioeconomic status by LGA and not at an individual level, so it is possible that our study is subject to ecological fallacy. For example, the LGAs are not homogenous so there will be people who are disadvantaged living in the least disadvantaged LGAs. In addition, it is possible that we did not have the power to detect many areas with rates that were significantly above or below the NSW rate, or areas that had a significantly temporal trend.

In summary, using a spatial temporal Bayesian model, has enabled us to obtain relative risk estimates for each LGA that incorporated spatial correlation, allowed the linear time trend to 
be different in each LGA and adjusted for LGA boundary changes. This would not have been possible with standard analysis techniques, commonly used in the literature. 


\section{FUNDING}

Robin Turner was employed as part of the New South Wales Biostatistical Officer Training Program funded by the New South Wales Department of Health while undertaking this work based at the New South Wales Injury Risk Management Research Centre. Caroline Finch was supported by a National Health and Medical Research Council Principal Research Fellowship. 


\section{REFERENCES}

1. Boufous S, Finch CF, Lord SR (2004) Incidence of hip fracture in New South Wales: are our efforts having an effect? Med J Aust 180:623-6.

2. Marks R, Allegrante JP, Ronald MacKenzie C, et al. (2003) Hip fractures among the elderly: causes, consequences and control. Ageing Research Reviews 2:57-93.

3. Kannus P, Niemi S, Parkkari J, et al. (1999) Hip fractures in Finland between 1970 and 1997 and predictions for the future.[see comment]. Lancet 353:802-5.

4. Balasegaram S, Majeed A, Fitz-Clarence H (2001) Trends in hospital admissions for fractures of the hip and femur in England, 1989-1990 to 1997-1998. J Public Health Med 23:11-7.

5. Rowe SM, Song EK, Kim JS, et al. (2005) Rising incidence of hip fracture in Gwangju City and Chonnam Province, Korea. J Korean Med Sci 20:655-8.

6. Yiannakoulias N, Rowe BH, Svenson LW, et al. (2003) Zones of prevention: the geography of fall injuries in the elderly. Soc Sci Med 57:2065-73.

7. Farahmand BY, Persson PG, Michaelsson K, et al. (2000) Socioeconomic status, marital status and hip fracture risk: a population-based case-control study. Osteoporos Int 11:803-8.

8. West J, Hippisley-Cox J, Coupland CA, et al. (2004) Do rates of hospital admission for falls and hip fracture in elderly people vary by socio-economic status? Public Health 118:576-81.

9. Brennan nee Saunders J, Johansen A, Butler J, et al. (2003) Place of residence and risk of fracture in older people: a population-based study of over 65-year-olds in Cardiff. Osteoporos Int 14:515-9. 
10. MacNab YC. (2004) Bayesian spatial and ecological models for small-area accident and injury analysis. Accid Anal Prev 36:1019-28.

11. Meliker JR, Maio RF, Zimmerman MA, et al. (2004) Spatial analysis of alcoholrelated motor vehicle crash injuries in southeastern Michigan. Accid Anal Prev 36:1129-35.

12. Nkhoma ET, Ed Hsu C, Hunt VI, et al. (2004) Detecting spatiotemporal clusters of accidental poisoning mortality among Texas counties, U.S., 1980 - 2001. Int J Health Geogr 3:25.

13. Australian Standard Geographical Classification (ASGC), ABS Catalogue Number 1216.0, www.abs.gov.au.

14. Lawson AB, Browne WJ, Vidal Rodeiro CL (2003) Disease Mapping with WinBUGS and MlwiN. John Wiley \& Sons Ltd, England.

15. Gelman A, Carlin JB, Stern HS et al. (2003) Bayesian Data Analysis, 2nd edition. Chapman and Hall.

16. Boufous S, Finch C, Close J et al. (2007) Hospital admissions following presentations to emergency departments for a fracture in older people. Injury Prevention. 13:211-214.

17. Sugarman JR, Connell FA, Hansen A et al. (2002) Hip Fracture Incidence in Nursing Home Residents and Community-Dwelling Older People, Washington State, 19931995. Journal of the American Geriatrics Society 50:1638-1643.

18. Gillespie LD, Gillespie WJ, Robertson MC et al. (2003) Interventions for preventing falls in elderly people. The Cochrane Database of Systematic Reviews Issue 4 Art No.: CD000340. DOI: 10.1002/14651858.CD000340 (accessed May 2006).

19. McClure R, Turner C, Peel N et al. (2005) Population-based interventions for the prevention of fall-related injuries in older people. The Cochrane Database of 
Systematic Reviews Issue 1. Art. No.: CD004441.pub2. DOI:

10.1002/14651858.CD004441.pub2 (accessed May 2006).

20. Butler M, Norton R, Lee-Joe T et al. (1996) The risks of hip fracture in older people from private homes and institutions. Age and Ageing 25:381-385. 


\section{TABLES}

Table 1: Age and sex standardised hip fracture admission ratios (95\% credible intervals) in New South Wales residents aged 65+ years, 07/98-06/04.

\begin{tabular}{|c|c|c|}
\hline Covariate & Definition & $\begin{array}{l}\text { Standardised Admission Ratio (SAR) } \\
\text { (95\% Credible Interval) }\end{array}$ \\
\hline
\end{tabular}

\begin{tabular}{|c|c|c|}
\hline Residential Aged Care Facility & The number of older persons in & 1.003 \\
\hline \multirow[t]{2}{*}{ (per 1000) } & residential aged care facilities per 1000 & $(1.002,1.004)$ \\
\hline & of the population aged $65^{+}$ & \\
\hline \multirow[t]{2}{*}{ SES Quintile 1} & The most disadvantaged LGAs with & 0.930 \\
\hline & approximately $1 / 5$ of the population & $(0.794,1.090)$ \\
\hline \multirow[t]{2}{*}{ SES Quintile 2} & & 0.890 \\
\hline & & $(0.770,1.036)$ \\
\hline \multirow[t]{2}{*}{ SES Quintile 3} & & 0.837 \\
\hline & & $(0.717,0.972)$ \\
\hline \multirow[t]{2}{*}{ SES Quintile 4} & & 0.855 \\
\hline & & $(0.743,0.989)$ \\
\hline \multirow[t]{2}{*}{ SES Quintile 5 (Reference Group) } & The least disadvantaged LGAs making & 1.000 \\
\hline & up approximately $1 / 5$ of the population & \\
\hline
\end{tabular}




\begin{tabular}{lclc|}
\hline \multicolumn{3}{l}{$\begin{array}{l}\text { Table 2: The local government areas with the five highest and five lowest } \\
\text { standardised admission ratios. }\end{array}$} \\
$\begin{array}{l}\text { Local Government } \\
\text { Areas with Highest }\end{array}$ & $\begin{array}{c}\text { Standardised } \\
\text { Admission Ratio } \\
\text { (SAR) } \\
\text { (95\% Credible Interval) }\end{array}$ & $\begin{array}{c}\text { Local Government } \\
\text { Areas with Lowest }\end{array}$ & $\begin{array}{c}\text { Standardised } \\
\text { Admission Ratio } \\
\text { (SAR) } \\
\text { (95\% Credible Interval) }\end{array}$ \\
\hline Sydney & 1.669 & Richmond Valley & 0.347 \\
& $(1.186,2.322)$ & & $(0.267,0.434)$ \\
Broken Hill \& & 1.540 & Maclean & 0.515 \\
Unincorporated Far & $(1.275,1.838)$ & & $(0.382,0.663)$ \\
West & 1.507 & Copmanhurst & 0.549 \\
Mosman & $(1.241,1.815)$ & & $(0.377,0.762)$ \\
& 1.439 & Muswellbrook & 0.551 \\
Baulkham Hills & $(1.279,1.609)$ & Kyogle & $(0.379,0.752)$ \\
Berrigan & 1.433 & & 0.557 \\
& $(1.108,1.840)$ & & $(0.394,0.755)$ \\
\hline
\end{tabular}




\section{FIGURE LEGENDS}

Figure 1. Smoothed relative risk for hospitalized hip fracture for each local government area (LGA) in New South Wales, Australia from July 2003 to June 2004, adjusted for changes to the LGA boundaries.

Figure 2. Smoothed relative risk for hospitalized hip fracture for each local government area (LGA) in the Sydney region of eastern New South Wales, Australia from July 2003 to June 2004, adjusted for changes to the LGA boundaries.

Figure 3. Smoothed relative risk for hospitalized hip fracture for each local government area (LGA) where the rate was significantly higher than the state average in New South Wales, Australia from July 2003 to June 2004, adjusted for changes to the LGA boundaries.

Figure 4. Smoothed relative risk for hospitalized hip fracture for each local government area (LGA) where the rate was significantly higher than the state average in the Sydney region of eastern New South Wales, Australia from July 2003 to June 2004, adjusted for changes to the LGA boundaries. 


\section{Statistical Appendix}

\section{Case Selection Criteria}

We investigated our case selection criteria through the use of a linked dataset, in which a unique identifier allows all episodes of care for an individual to be identified. We selected all episodes of care with a principal diagnosis code of hip fracture (S72.0-S72.2) and first external code of a fall (W00-W19) for the period July 2003 to June 2004 for period aged 65+ years. Our aim was to see whether our case selection criteria - ie excluding episodes of care for which the source referral was a transfer from another hospital or a type change admission - would correctly identify an individual's first episode of care for a hip fracture.

\begin{tabular}{|l|l|l|l|l|}
\hline \multirow{3}{*}{ referral } & \multicolumn{4}{|l|}{ Linked dataset } \\
\hline & & First episode of & Other episode & Total \\
& & care & of care & \\
\cline { 2 - 5 } & Included & 4763 & 487 & 5250 \\
\cline { 2 - 5 } & Excluded & 537 & 889 & 1426 \\
\cline { 2 - 5 } & & 5300 & 1276 & 6676 \\
\hline
\end{tabular}

We found that of the 5300 first episodes of care for hip fracture, our case selection criterion was able to identify $4763 / 5300=89.9 \%$. Of the 1276 subsequent episodes of care, the criterion successfully excluded $889=69.7 \%$ of these. In total, the use of the case selection criterion would underestimate the number of first episodes of care for hip fractures by about $0.9 \%$. 
Bayesian Modelling

We fitted a Poisson regression model for the SARs across the six years for each of the 175 LGAs [14]. The model took the form $Y_{i j} \sim \operatorname{Poisson}\left(E_{i j} \theta_{i j}\right)$, where $\log \left(\theta_{i j}\right)=$ overall rate + spatial effect + uncorrelated heterogeneity + boundary change effect + covariates of interest + temporal effect; or put mathematically:

$$
\begin{aligned}
\log \left(\theta_{i j}\right)= & \alpha+u_{i}+v_{i}+\beta_{1} X_{1 i j}+\delta_{1} X_{1 i j}+\beta_{2} X_{2 i j}+\delta_{2} X_{2 i j}+\beta_{3} X_{3 i j}+\delta_{3} X_{3 i j} \\
& +\beta_{4} X_{4 i j}+\beta_{5} X_{5 i}+\beta_{6} X_{6 i}+\beta_{7} X_{7 i}+\beta_{8} X_{8 i}+\beta_{9} t_{j}+\delta_{i} t_{j},
\end{aligned}
$$

In this model, $\theta_{i j}$ represents the relative risk of hospitalized hip fracture for the ith LGA at the $j$ th year, and the term $\alpha$ denotes the overall relative risk. The terms $u_{i}$ and $v_{i}$ denote the spatial correlation term and uncorrelated heterogeneity, that is extra variation that is spatially uncorrelated, respectively. Spatial correlation typically means that rates are more similar in areas that are geographically closer. Using standard Bayesian approaches [14], it is assumed that $u_{i}$ has a conditional autoregressive prior distribution, so that

$u_{i} \mid u_{j}, j \neq i \sim N\left(\frac{1}{\sum_{j} w_{i j}} \sum_{j} w_{i j} u_{j}, \frac{\tau_{u}^{2}}{\sum_{j} w_{i j}}\right)$,

for weights $w_{i j}=1$ if $i$ and $j$ are adjacent and 0 otherwise. The term $\tau_{u}$ has a gamma prior distribution and accounts for the spatial correlation in the model by smoothing the estimates in a LGA using the information from adjacent neighbours. 
The term $\beta_{1} X_{1 i j}+\delta_{1} X_{1 i j}+\beta_{2} X_{2 i j}+\delta_{2} X_{2 i j}+\beta_{3} X_{3 i j}+\delta_{3} X_{3 i j}$ was used to represent the three boundary change terms $\left(X_{1}, X_{2}, X_{3}\right)$. This means that the boundary change is modelled as a step function at the time the boundary change occurs, using a step function rather than a gradual change with was considered appropriate given that the data is being modelled per year and we do not expect boundary changes to have an impact greater than the year they occurred in. Each boundary change term included an interaction with a conditionally autoregressive spatial term $\left(\delta_{1}, \delta_{2}, \delta_{3}\right)$, to allow the boundary change to be different in each LGA. This was necessary because only a small number of LGAs (twenty six) had boundary changes over the study period and this model allows each change to be different. In a similar manner, the regression coefficients for changes across time were included in the model as $\beta_{9} t_{j}+\delta_{i} t_{j}$ to allow for a different time trend in each LGA.

Finally, the term $\beta_{4} X_{4 i j}+\beta_{5} X_{5 i}+\beta_{6} X_{6 i}+\beta_{7} X_{7 i}+\beta_{8} X_{8 i}$ represents the covariates included in the model. The first term represents the number of residents in aged-care facilities by time and LGA. The next four terms model the effects of four levels of socioeconomic status when compared to the most disadvantaged quintile. In this particular formulation of the model, the relationship between the population in aged-care facilities, SES and the rate of hip fractures is assumed to not depend on LGA. All the $\beta$ 's in the model have normal prior distributions.

Using standard Bayesian approaches [14], the posterior distributions for the parameters in the model were sampled using Markov Chain Monte Carlo methods. A burn-in of 25,000 iterations was used to ensure stationarity for each chain. A further 20,000 iterations were then retained for the posterior samples. 